\title{
A novel partial de novo duplication of JARID2 gene causing a neurodevelopmental phenotype
}

\section{Liisa Viitasalo}

HUS Diagnostic Center, Division of Genetics and Clinical Pharmacology, Department of Clinical Genetics, University of Helsinki and Helsinki University Hospital, Helsinki, Finland https://orcid.org/00000002-4014-6755

\section{Kaisa Kettunen}

HUS Diagnostic Center, Division of Genetics and Clinical Pharmacology, Laboratory of Genetics, University of Helsinki and Helsinki University Hospital, Helsinki, Finland https://orcid.org/0000-00017402-461X

\section{Matti Kankainen}

HUS Diagnostic Center, Division of Genetics and Clinical Pharmacology, Laboratory of Genetics, University of Helsinki and Helsinki University Hospital, Helsinki, Finland https://orcid.org/0000-00024714-9481

\section{Elina H. Niemelä}

HUS Diagnostic Center, Division of Genetics and Clinical Pharmacology, Laboratory of Genetics, University of Helsinki and Helsinki University Hospital, Helsinki, Finland https://orcid.org/0000-00016871-6441

\section{Kirsi Kiiski ( $\nabla$ kirsi.kiiski@hus.fi )}

HUS Diagnostic Center, Division of Genetics and Clinical Pharmacology, Laboratory of Genetics, University of Helsinki and Helsinki University Hospital, Helsinki, Finland https://orcid.org/0000-00021004-9612

\section{Case Report}

Keywords: JARID2, duplication, de novo, pathogenic variant, whole genome sequencing, RNA sequencing

Posted Date: January 27th, 2022

DOI: https://doi.org/10.21203/rs.3.rs-1299950/v1

License: (c) (i) This work is licensed under a Creative Commons Attribution 4.0 International License. Read Full License 
Title

\section{A novel partial de novo duplication of JARID2 gene causing a neurodevelopmental phenotype}

\section{Running title}

A novel partial de novo duplication of JARID2

\section{Authors}

Liisa Viitasalo ${ }^{1}$, Kaisa Kettunen ${ }^{2}$, Matti Kankainen ${ }^{2}$, Elina H. Niemelä ${ }^{2}$, Kirsi Kiiski ${ }^{2}$

${ }^{1}$ HUS Diagnostic Center, Division of Genetics and Clinical Pharmacology, Department of Clinical Genetics, University of Helsinki and Helsinki University Hospital, Helsinki, Finland

${ }^{2}$ HUS Diagnostic Center, Division of Genetics and Clinical Pharmacology, Laboratory of Genetics, University of Helsinki and Helsinki University Hospital, Helsinki, Finland

\section{Acknowledgements}

We would like to thank the family for taking part in this research. The authors thank Hanna Tammio for excellent technical assistance and MD PhD Kristiina Avela for great support in clinical evaluations. We also thank the Core facility Sequencing Unit at FIMM Technology Centre supported by University of Helsinki and Biocenter Finland.

Funding: Special Funding for University level Health Research (Kiiski, Viitasalo, Kettunen, Kankainen).

This study makes use of data generated by the DECIPHER community. Those who carried out the original analysis and collection of the data bear no responsibility for the further analysis or interpretation of the results in this paper. A full list of centres who contributed to the generation of the data is available from https://deciphergenomics.org/about/stats.

\section{Conflict of Interest Statement}

The authors have no conflict of interest to report.

\section{Data Availability Statement}

The patient phenotype and duplication data has been submitted to the DECPIHER database (ID: 345282) (Firth et al., 2009). Genomic sequencing read data are available from the corresponding authors with permission of the Helsinki and Uusimaa Hospital district Ethics Committee. National and institutional 
regulations prohibit deposition of these data in public repositories. The authors confirm that data supporting the findings of this study are available within the article and its supplementary materials.

\section{Abstract}

Deletions covering the entire or partial JARID2 gene as well as pathogenic single nucleotide variants leading to haploinsufficiency of JARID2 have recently been shown to cause a clinically distinct neurodevelopmental syndrome phenotype. Here, we present a previously undescribed partial de novo duplication of the JARID2 gene in a patient displaying a phenotype associated with known JARID2 loss-of-function variant carriers. The phenotype of the index patient included coordination problems, clumsiness, language delay, unclear speech, problems with behavior and attention as well as difficulties with social contacts and daily activities. The patient has markedly dark infraorbital circles and slightly prominent supraorbital ridges. The phenotype shares a notable resemblance to previously characterized JARID2 deletion patients. Genetic analyses from the samples of the index patient and the parents were performed. Whole-genome sequencing and array comparative genomic hybridization revealed a novel disease-causing variant type, a partial tandem duplication of JARID2, covering the exons 1-7. Furthermore, RNA sequencing validated increased expression of these exons. Expression alterations were also detected in the target genes of PRC2 complex, in which JARID2 acts as an essential member. Our data adds to the variety of different pathogenic variants causing the JARID2 specific neurodevelopmental syndrome phenotype.

\section{Keywords:}

JARID2, duplication, de novo, pathogenic variant, whole genome sequencing, RNA sequencing

\section{Introduction}

The JARID2 gene (Jumonji and AT-rich interaction domain 2, OMIM *601594) encodes an ARID transcription factor that is widely detected across human tissues (Bergé-Lefranc et al., 1996). The protein is localized in the nucleoplasm and mitochondria of the cells (Peng et al., 2009) and contains the Jumonji N (JmjN), AT-rich interaction domain (ARID), Jumonji C (JmjC), and zinc finger domains (Cooper et al., 2016; Takeuchi et al., 2006). Unlike in the other Jumonji family proteins, the JmjC domain of JARID2 lacks its histone demethylase activity (Cooper et al., 2016; Takeuchi et al., 2006). Instead, JARID2 interacts with the Polycomb repressive complex 2 (PRC2) that is critical for lineage commitment during embryonic development and maintenance of cell type identity. In the complex, JARID2 contributes to the recruitment of PRC2 to chromatin (Peng et al., 2009) and regulation of histone H3 lysine 27 (H3K27) methylation activity of PRC2 (Son et al., 2013). The 
region containing amino acids 119-574, corresponding to exons 4 to 7 , appears to mediate these activities (Cooper et al., 2016; Li et al., 2010; Son et al., 2013). Highlighting the crucial role of JARID2, its deletion in mice results in severe abnormalities in multiple organs, neural defects and mid-late gestation lethality (Takeuchi et al., 1995, 2006). During embryogenesis, JARID2 is predominantly expressed in neurons and is also highly expressed in the adult cerebellum (Bergé-Lefranc et al., 1996; Takeuchi et al., 1995).

In humans, heterozygous deletions containing entire or partial JARID2 gene have been characterized and associated with several congenital defects (Barøy et al., 2013; Verberne et al., 2021). JARID2 is located within the $6 p 22$ microdeletion region and two SRO regions (smallest regions of overlap) have been identified. SRO I contains JARID2 and DTNBP1 whereas SRO || GMPR and ATXN1 genes. Of those, chromatin modifier genes JARID2 and ATXN1 were suggested as likely candidate disease causing genes (Barøy et al., 2013).

Recently, a study including 16 patients from 15 families with different JARID2 disease-causing variants was published characterizing one whole-gene and 7 intragenic deletions as well as small variant alternations including 2 frameshift, 2 nonsense, one splice-site and 3 missense variants. All variants were predicted to lead to JARID2 haploinsufficiency due to nonsense-mediated mRNA decay (Verberne et al., 2021). Haploinsufficiency of JARID2 has been shown to cause a clinically distinct neurodevelopmental phenotype (Barøy et al., 2013; Verberne et al., 2021). The typical features of patients with JARID2 haploinsufficiency include developmental delay or intellectual disability, autistic features, behavioral abnormalities and mild dysmorphic features such as deep-set eyes and infraorbital dark circles, prominent supraorbital ridges and midface hypoplasia (Barøy et al., 2013; Di Benedetto et al., 2013; Verberne et al., 2021). Duplications and deletions of exon 6 have been identified in patients with intellectual disability as well as in control population and these have been interpreted as benign variation (Tucker et al., 2014; Zahir et al., 2016).

In addition to the present case, DECIPHER database recognizes three other patients with a partial JARID2 duplication. Two of these have 569 and $576 \mathrm{~kb}$ duplications of unknown origin covering only the first exon of JARID2. The first-mentioned (ID:260089) has also a second $278 \mathrm{~kb}$ microduplication harboring the exons 4-5 of PTPRD gene (NM_002839.4). The phenotypic features include intellectual disability, macrocephaly and myoclonus. The latter patient (ID:267637) has also a de novo heterozygous missense variant c.902A>G, p.(Tyr301Cys) in GABRB2. The phenotypic features include blepharophimosis, broad hallux, frontal bossing, generalized hypotonia, generalized myoclonic and photosensitive tonic-clonic seizures, global developmental delay, kyphosis, large earlobe, macrocephaly and sparse scalp hair. The third patient (ID:255516) has inherited a duplication of JARID2 exons 1-2 from an unaffected parent. The reported phenotypic features are atrioventricular canal defect, poor speech and short stature. 
Here, a comprehensive genetic analysis revealed the presence of a previously uncharacterized partial de novo duplication of the JARID2 gene as the sole finding in a patient displaying a phenotype associated with known JARID2 loss-of-function variant carriers.

\section{Materials and Methods}

See Supplementary Data 1A-D and Supplementary Tables 1-3.

\section{Results}

\section{Genetic analyses}

Various complementing whole-genome and targeted assays were used to discover and validate the presence of a partial tandem duplication of JARID2 (Figure 1).

First, a whole genome chromosomal microarray (Figure 1, Supplementary Data 1A, Supplementary Table 1) revealed a partial duplication of the JARID2 gene, in chromosomal region 6p23p22.3, the size being between 309-346 kb. This duplication GRCh38 chr6:g.(15158120_15181642)_(15490178_15504505)dup encompassed at least exons 1-6 of the 18 exons of JARID2 (NM_004973.4). The status of exons 7 and 8 remained uninformative. The index patient had no other clinically relevant copy number variants and the parental array-CGH profiles were normal.

A fluorescent in situ hybridization (FISH) study (Figure 1, Supplementary Data 1B) targeting the duplicated region was consistent with a tandem duplication. However, the orientation of the duplication remained unelucidated. The parental FISH tests showed normal results, excluding insertional translocation in the parents and corroborating the de novo status of the duplication.

Whole genome sequencing (WGS) (Figure 1, Supplementary Data 1C, Supplementary Tables 1-2) defined the breakpoint to intron 7 (NM_004973.4); GRCh38 chr6:g.15160000-15499999dup. Thus, the duplicated exons were 1-7, which contain the JmjN domain and part of the ARID/BRIGHT DNA binding domain. The duplicated allele was of maternal origin. The read pair orientation analysis indicated a tandem duplication, but because of the repetitive nature of the genomic regions around the breakpoints, definitive conclusions could not be established.

RNA sequencing analysis (Figure 1, Supplementary Data 1D, Supplementary Tables 2-3) indicated that the RNA expression levels are elevated for exons 1-7 of JARID2, suggesting that the $\mathrm{N}$-terminal fragment is expressed as an isolated transcript. This finding could not be confirmed on protein level due to lack of patient 
material. Using a z-score test and 95 percent confidence level, 345 genes (61\%) were downregulated and 225 (39\%) were upregulated in the patient compared to his mother (Supplementary Table 3). This suggests that the duplication of the $\mathrm{N}$-terminal domain of JARID2 results in a global transcriptional repression. Many target genes of JARID2 (Peng et al., 2009), showed altered expression in the patient (p-value 0.027). These included genes involved in neuronal development, neuronal function and cellular differentiation, for example MDGA1, SOX6, TMTC1, ATOH8, TSPAN5, CACNA1I, EGR2, KLHL31, GATA6, and EGR3.

\section{Clinical report}

The study was approved by the Ethical Review Board of Helsinki University hospital (233/13/03/00/11) and was performed in accordance with the Declaration of Helsinki of 1975. The parents gave informed written consent.

The phenotypic features of the index patient as well as the previously characterized patients (Barøy et al., 2013; Verberne et al., 2021) are presented in Table 1.

The index patient is the second child to nonconsanguineous Finnish parents. The pregnancy and delivery were uneventful. He was born at 40 weeks and 5 days of gestation with a birth weight of 4,280 g (+55 percentiles), length $53 \mathrm{~cm}(+1 \mathrm{SD})$ and occipitofrontal circumference $36 \mathrm{~cm}(+0.5 \mathrm{SD})$. The Apgar scores were 7, 9 and 10 at 1, 5 and 10 minutes, respectively. He had mild jaundice and slightly elevated bilirubin levels that normalized quickly without treatment. He started walking at the age of one year. According to the parents, he had problems with coordination and clumsiness since the first years of life. He spoke his first words at 2 years of age and small sentences (2-3 words) at 2.5 years. He had eartubes inserted at the age of two years because of glue ear. At 3.5 years of age, he was referred to a phoniatrist due to language delay and unclear speech. Submucous cleft palate was observed. Speech therapy was started, which helped him reach the age-appropriate level. However, he continued to have problems with articulation. Ophthalmologist prescribed glasses for strabismus.

At the age of six, the cognitive level of the patient was estimated to be normal. When starting school, he needed extra support in learning to read. He had problems with attention and behavior and was transferred to a smaller group to help him focus on class. Later, he started in a hospital elementary school with an adjusted curriculum. He had difficulties with tolerating adversities and often threw tantrums. He also had problems with social contacts. At 10 years of age, he was referred to children's neuropsychiatric clinic and was diagnosed with attention deficit hyperactivity disorder (ICD code f90.0), other disorders of psychological development (f88) with specific neurocognitive and Asperger features. Methylfenidate alleviated the 
symptoms to some extent. Neuropsychiatric therapy was started and continued for several years. He also received occupational therapy and physiotherapy for posture problems.

When moving to junior high school the patient attended a normal curriculum. At his last evaluation in 2020, he had progressed in many daily activities and improved his social skills but continues to have challenges with attention and interaction. His grades have been on a mediocre level.

The patient has grown approximately on a +2.5 SD curve, the expected height being +0.6 SD. When last measured his weight was +30 percentiles $(>2 S D$ ). The patient's facial features include markedly dark infraorbital circles, mild ptosis, slightly prominent supraorbital ridges, mild midface hypoplasia and full earlobes and lips. He has pes planus and syndactyly between II and III toes. Mild balance and coordination problems and slightly low muscle tone were observed in last neurological status.

\section{Discussion}

We present a previously undescribed partial de novo duplication of the JARID2 gene in a patient sharing phenotypic features with previously characterized patients with JARID2 haploinsufficiency. According to our knowledge, this is the first characterized case of JARID2 duplication with altered expression of the duplicated region exons 1-7. The data suggests that the defective transcripts are not entirely degraded by nonsensemediated decay, unlike reported for previously described variants (Verberne et al., 2021).

To investigate the effect of the duplication of our index patient on the transcriptional regulatory role of JARID2, we performed to our knowledge the first transcriptomic analysis of JARID2 variants in human subjects. We discovered that the exons 1-7, present in the partial duplication, were expressed in excess on RNA level. We also noticed that more genes were downregulated in the patient in comparison to his mother, and among these dysregulated genes were several JARID2 target genes identified by ChIP-seq (Peng et al., 2009). These genes are involved in neuronal function and differentiation and the majority of them were downregulated in the patient. The widespread downregulation was unexpected as the patient's phenotype closely resembles that of the previously described JARID2 haploinsufficiency cases. Loss of PRC2 components typically results in reduced H3K27me3 mark, and consequently, derepression of PRC2 target genes (Azuara et al., 2006; Boyer et al., 2006; Lee et al., 2006; Shen et al., 2008). Previous studies on the function of an isolated $\mathrm{N}$-terminal domain also suggest that the domain enhances the recruitment of PRC2 to chromatin and stimulates its methyltransferase activity (Cooper et al., 2016; Li et al., 2010; Son et al., 2013). On the other hand, full-length Jarid2 deficiency in mouse ES cells has been shown to reduce the expression of PRC2 target genes via impaired recruitment of PRC1 and RNA polymerase II (Landeira et al., 2010). Given the close resemblance of the patient's phenotype to JARID2 haploinsufficient patients, our data suggests that the 
duplication of exons 1-7 results in loss of function in JARID2 protein and reduced expression of PRC2 target genes.

Compared to the previously reported cases, our index patient showed majority of the most common phenotypic features (Table 1) (Barøy et al., 2013; Verberne et al., 2021). These include developmental delay (speech delay), features of autism spectrum disorder and behavioral problems as well as the notably dark infraorbital circles that were observed in $29 \%$ of previous cases, height and weight were $>2$ SD as in a substantial proportion of previous cases ( $31 \%$ and $25 \%$, respectively). As much as $47 \%$, including the present patient, had perinatal complications, however, majority were rather mild and common findings such as hyperbilirubinemia and thus possibly not related to the JARID2 variants. He also presented with syndactyly whereas $39 \%$ of patients showed hand/foot anomalies. However, these characteristics are also rather frequent and unspecific. In contrast to all the previous cases, the current patient does not present with intellectual disability, and despite continuing challenges, he has attended to normal curriculum.

Three other patients with a partial JARID2 duplication in the DECIPHER database (ID:s 260089, 267637 and 255516) share some of the clinical features with our index case and the patients described in the literature, such as developmental delay, intellectual disability, macrocephaly and frontal bossing. The characterized patients present with a rather wide spectrum of features, making it difficult to evaluate the genotypephenotype correlation. In addition, two of these DECIPHER patients have a second genetic variant, which further complicates the evaluation. The only previously characterized patient with JARID2 partial duplication as the sole finding, had a smaller duplication, spanning only the first two exons of JARID2. As opposed to the majority of previously described patients with de novo variants, this patient had inherited the duplication from an unaffected parent, which increases the uncertainty of the effect of this particular duplication (Table 1). Many factors, such as the location and exact breakpoints of the duplications, are likely to affect their pathogenicity and thus more data would be required to evaluate the significance of the duplications reported in DECIPHER.

In conclusion, we present a de novo JARID2 duplication in a patient whose clinical presentation significantly overlaps with formerly characterized patients with JARID2 haploinsufficiency. Our findings suggest, that in addition to haploinsufficiency, other molecular mechanisms might lead to JARID2-associated condition. Since the neurocognitive capacity of the current patient is higher than in patients with whole gene deletions and JARID2 variants predicted to undergo nonsense-mediated decay, partial duplications might be associated with somewhat milder phenotype. However, functional studies are needed to demonstrate the molecular mechanisms and the associated phenotypes of different JARID2 variants. 


\section{References}

Azuara, V., Perry, P., Sauer, S., Spivakov, M., Jørgensen, H. F., John, R. M., Gouti, M., Casanova, M., Warnes, G., Merkenschlager, M., \& Fisher, A. G. (2006). Chromatin signatures of pluripotent cell lines. Nature Cell Biology, 8(5), 532-538. https://doi.org/10.1038/ncb1403

Barøy, T., Misceo, D., Strømme, P., Stray-Pedersen, A., Holmgren, A., Rødningen, O. K., Blomhoff, A., Helle, J. R., Stormyr, A., Tvedt, B., Fannemel, M., \& Frengen, E. (2013). Haploinsufficiency of two histone modifier genes on 6p22.3, ATXN1 and JARID2, is associated with intellectual disability. Orphanet Journal of Rare Diseases, 8(1). https://doi.org/10.1186/1750-1172-8-3

Bergé-Lefranc, J. L., Jay, P., Massacrier, A., Cau, P., Mattei, M. G., Bauer, S., Marsollier, C., Berta, P., \& Fontes, M. (1996). Characterization of the human jumonji gene. Human Molecular Genetics, 5(10). https://doi.org/10.1093/hmg/5.10.1637

Boyer, L. A., Plath, K., Zeitlinger, J., Brambrink, T., Medeiros, L. A., Lee, T. I., Levine, S. S., Wernig, M., Tajonar, A., Ray, M. K., Bell, G. W., Otte, A. P., Vidal, M., Gifford, D. K., Young, R. A., \& Jaenisch, R. (2006). Polycomb complexes repress developmental regulators in murine embryonic stem cells. Nature, 441(7091), 349-353. https://doi.org/10.1038/nature04733

Cooper, S., Grijzenhout, A., Underwood, E., Ancelin, K., Zhang, T., Nesterova, T. B., Anil-Kirmizitas, B., Bassett, A., Kooistra, S. M., Agger, K., Helin, K., Heard, E., \& Brockdorff, N. (2016). Jarid2 binds monoubiquitylated H2A lysine 119 to mediate crosstalk between Polycomb complexes PRC1 and PRC2. Nature Communications, 7. https://doi.org/10.1038/ncomms13661

Di Benedetto, D., Di Vita, G., Romano, C., Lo Giudice, M., Vitello, G. A., Zingale, M., Grillo, L., Castiglia, L., Musumeci, S. A., \& Fichera, M. (2013). 6p22.3 deletion: report of a patient with autism, severe intellectual disability and electroencephalographic anomalies. Molecular Cytogenetics, 6(1). https://doi.org/10.1186/1755-8166-6-4

Firth, H. V, Richards, S. M., Bevan, A. P., Clayton, S., Corpas, M., Rajan, D., Van Vooren, S., Moreau, Y., Pettett, R. M., \& Carter, N. P. (2009). DECIPHER: Database of Chromosomal Imbalance and Phenotype in Humans Using Ensembl Resources. American Journal of Human Genetics, 84(4), 524-533. https://doi.org/10.1016/j.ajhg.2009.03.010

Landeira, D., Sauer, S., Poot, R., Dvorkina, M., Mazzarella, L., Jørgensen, H. F., Pereira, C. F., Leleu, M., Piccolo, F. M., Spivakov, M., Brookes, E., Pombo, A., Fisher, C., Skarnes, W. C., Snoek, T., Bezstarosti, K., Demmers, J., Klose, R. J., Casanova, M., ... Fisher, A. G. (2010). Jarid2 is a PRC2 component in embryonic stem cells required for multi-lineage differentiation and recruitment of PRC1 and RNA Polymerase II to developmental regulators. Nature Cell Biology, 12(6), 618-624. https://doi.org/10.1038/ncb2065

Lee, T. I., Jenner, R. G., Boyer, L. A., Guenther, M. G., Levine, S. S., Kumar, R. M., Chevalier, B., Johnstone, S. E., Cole, M. F., Isono, K., Koseki, H., Fuchikami, T., Abe, K., Murray, H. L., Zucker, J. P., Yuan, B., Bell, G. W., Herbolsheimer, E., Hannett, N. M., ... Young, R. A. (2006). Control of Developmental Regulators by Polycomb in Human Embryonic Stem Cells. Cell, 125(2), 301-313. https://doi.org/https://doi.org/10.1016/j.cell.2006.02.043

Li, G., Margueron, R., Ku, M., Chambon, P., Bernstein, B. E., \& Reinberg, D. (2010). Jarid2 and PRC2, partners in regulating gene expression. Genes and Development, 24(4). https://doi.org/10.1101/gad.1886410

Peng, J. C., Valouev, A., Swigut, T., Zhang, J., Zhao, Y., Sidow, A., \& Wysocka, J. (2009). Jarid2/Jumonji Coordinates Control of PRC2 Enzymatic Activity and Target Gene Occupancy in Pluripotent Cells. Cell, 139(7). https://doi.org/10.1016/j.cell.2009.12.002

Shen, X., Liu, Y., Hsu, Y.-J., Fujiwara, Y., Kim, J., Mao, X., Yuan, G.-C., \& Orkin, S. H. (2008). EZH1 Mediates 
Methylation on Histone H3 Lysine 27 and Complements EZH2 in Maintaining Stem Cell Identity and Executing Pluripotency. Molecular Cell, 32(4), 491-502.

https://doi.org/https://doi.org/10.1016/j.molcel.2008.10.016

Son, J., Shen, S. S., Margueron, R., \& Reinberg, D. (2013). Nucleosome-binding activities within JARID2 and EZH1 regulate the function of PRC2 on chromatin. Genes and Development, 27(24). https://doi.org/10.1101/gad.225888.113

Takeuchi, T., Watanabe, Y., Takano-Shimizu, T., \& Kondo, S. (2006). Roles of jumonji and jumonji family genes in chromatin regulation and development. Developmental Dynamics : An Official Publication of the American Association of Anatomists, 235(9), 2449-2459. https://doi.org/10.1002/dvdy.20851

Takeuchi, T., Yamazaki, Y., Katoh-Fukui, Y., Tsuchiya, R., Kondo, S., Motoyama, J., \& Higashinakagawa, T. (1995). Gene trap capture of a novel mouse gene, jumonji, required for neural tube formation. Genes and Development, 9(10). https://doi.org/10.1101/gad.9.10.1211

Tucker, T., Zahir, F. R., Griffith, M., Delaney, A., Chai, D., Tsang, E., Lemyre, E., Dobrzeniecka, S., Marra, M., Eydoux, P., Langlois, S., Hamdan, F. F., Michaud, J. L., \& Friedman, J. M. (2014). Single exon-resolution targeted chromosomal microarray analysis of known and candidate intellectual disability genes.

European Journal of Human Genetics, 22(6). https://doi.org/10.1038/ejhg.2013.248

Verberne, E. A., Goh, S., England, J., van Ginkel, M., Rafael-Croes, L., Maas, S., Polstra, A., Zarate, Y. A., Bosanko, K. A., Pechter, K. B., Bedoukian, E., Izumi, K., Chaudhry, A., Robin, N. H., Boothe, M., Lippa, N. C., Aggarwal, V., De Vivo, D. C., Lehman, A., ... Campeau, P. M. (2021). JARID2 haploinsufficiency is associated with a clinically distinct neurodevelopmental syndrome. Genetics in Medicine, 23(2). https://doi.org/10.1038/s41436-020-00992-z

Zahir, F. R., Tucker, T., Mayo, S., Brown, C. J., Lim, E. L., Taylor, J., Marra, M. A., Hamdan, F. F., Michaud, J. L., \& Friedman, J. M. (2016). Intragenic CNVs for epigenetic regulatory genes in intellectual disability: Survey identifies pathogenic and benign single exon changes. American Journal of Medical Genetics Part A, 170(11), 2916-2926. https://doi.org/https://doi.org/10.1002/ajmg.a.37669 
Table 1. A review of the molecular genetic and clinical findings of index patient and presently published patients with JARID2 variants.

\begin{tabular}{|c|c|c|c|c|c|c|c|c|c|c|c|c|c|c|c|c|c|c|c|}
\hline $\begin{array}{l}1=\text { Baroy et al. } 2013 \\
2=\text { Verbene et al. } 2020\end{array}$ & 1 & 2 & 2 & 2 & 2 & 2 & 2 & 2 & 2 & 2 & 2 & 2 & 2 & 2 & 2 & 2 & 2 & $\begin{array}{l}\text { Index } \\
\text { patient }\end{array}$ & $\begin{array}{c}\text { Proportion } \\
\text { of positive } \\
\text { findings (\%) }\end{array}$ \\
\hline Gender & $\mathrm{M}$ & $\mathrm{F}$ & $\mathrm{F}$ & $\mathrm{M}$ & $\mathrm{M}$ & $\mathrm{F}$ & $\mathrm{M}$ & $\mathrm{M}$ & $\mathrm{M}$ & $\mathrm{M}$ & $\mathrm{M}$ & $\mathrm{M}$ & $\mathrm{M}$ & $\mathrm{M}$ & $\mathrm{M}$ & $\mathrm{M}$ & $\mathrm{F}$ & $\mathrm{M}$ & \\
\hline Age at last follow-up (y) & 6.5 & 17 & 19 & 9 & 3.5 & 7 & 38 & 4 & 10 & 12.5 & 7.3 & 23 & 4 & 3.2 & 8 & 39 & 10.8 & 12 & \\
\hline \multicolumn{20}{|l|}{ Genetic variant } \\
\hline Variant type & del & del & del & del & del & del & del & del & del & fs & $\mathrm{ns}$ & fs & $\mathrm{ns}$ & ss & $\mathrm{ms}$ & $\mathrm{ms}$ & $\mathrm{ms}$ & dup & \\
\hline Size $(k b)$ & 189 & 30 & 90 & 100 & 120 & 140 & 140 & 205 & 320 & & & & & & & & & 309 & \\
\hline Exons & & 2 & $2-3$ & 2 & $2-3$ & $2-5$ & $2-5$ & $1-2$ & $1-18$ & 13 & 8 & 16 & 16 & \begin{tabular}{|l|l}
11 \\
\end{tabular} & 4 & 8 & 7 & $1-7$ & \\
\hline Inheritance & $\mathrm{dn}$ & $\mathrm{dn}$ & $\mathrm{dn}$ & $\mathrm{dn}$ & $\mathrm{dn}$ & $p$ & na & na & $\mathrm{dn}$ & $\mathrm{dn}$ & na & $\mathrm{dn}$ & $\mathrm{dn}$ & $\mathrm{dn}$ & $\mathrm{dn}$ & $\mathrm{dn}$ & $\mathrm{dn}$ & & \\
\hline \multicolumn{20}{|l|}{ Growth } \\
\hline Height > 2 SD & - & - & + & - & - & + & na & + & - & + & - & - & - & - & + & - & - & + & 35 \\
\hline Weight $>2$ SD & - & - & - & + & - & - & na & + & - & - & - & - & - & - & + & - & + & + & 29 \\
\hline Head circumference $>2$ SD & - & - & na & na & - & - & na & + & - & - & - & na & - & - & + & - & - & - & 14 \\
\hline \multicolumn{20}{|l|}{ Facial features } \\
\hline Deep set eyes & + & + & + & - & - & + & + & - & - & - & - & - & + & + & - & - & - & + & 44 \\
\hline Full lips & + & + & - & - & + & - & - & - & - & + & + & + & - & - & - & - & - & + & 39 \\
\hline High anterior hairline & na & + & + & - & + & + & + & - & - & + & - & - & - & - & - & - & - & - & 35 \\
\hline Infraorbital dark circles & + & + & - & + & - & + & + & - & - & - & - & - & - & - & - & - & - & + & 33 \\
\hline Prominent supraorbital ridges & + & - & - & - & - & + & + & - & - & - & - & + & - & - & - & - & - & + & 28 \\
\hline Deep set nasal root & + & + & - & - & + & + & + & - & - & - & - & - & - & - & - & - & - & - & 28 \\
\hline Midface hypoplasia & + & - & + & - & - & + & + & - & - & - & - & - & - & - & - & - & - & + & 28 \\
\hline Bulbous nasal tip & + & + & - & - & - & + & + & + & - & - & - & - & - & - & - & - & - & - & 28 \\
\hline Short philtrum & + & + & + & - & - & - & - & - & - & + & - & - & - & - & - & - & - & - & 22 \\
\hline Broad forehead & - & - & - & + & - & + & + & - & - & - & - & - & - & + & - & - & - & - & 22 \\
\hline \multicolumn{20}{|l|}{ Neurology } \\
\hline Developmental delay & + & + & + & + & + & + & + & + & + & + & + & + & + & + & + & + & + & + & 100 \\
\hline Intellectual disability & - & + & - & + & - & + & + & + & - & + & + & + & + & na & + & - & + & - & 65 \\
\hline ASD features & - & + & - & + & + & - & - & + & - & + & + & - & + & - & + & + & - & + & 55 \\
\hline Behavioral abnormalities & - & - & + & - & - & - & - & + & - & + & + & + & - & - & + & + & - & + & 44 \\
\hline Abnormal MRI & - & na & na & na & - & - & na & - & - & + & na & - & + & + & + & na & na & na & 40 \\
\hline Hypotonia & + & - & - & - & + & - & - & + & - & - & + & - & + & + & - & - & - & - & 33 \\
\hline Eye/vision abnormalities & + & - & - & + & - & - & - & - & - & - & + & - & - & - & + & + & - & + & 33 \\
\hline ASD diagnosis & - & - & - & - & - & - & - & - & - & + & - & - & - & - & + & + & - & - & 17 \\
\hline Epilepsy & - & - & - & - & - & - & + & + & - & - & - & + & - & - & - & - & - & - & 17 \\
\hline Gait disturbance & + & - & - & - & - & - & - & - & - & - & - & - & + & - & - & - & - & - & 11 \\
\hline \multicolumn{20}{|l|}{ Other } \\
\hline Perinatal complications & - & - & - & + & + & - & + & - & - & + & na & - & + & - & + & + & - & + & 47 \\
\hline Hand/foot anomalies & + & + & - & + & - & + & - & + & - & - & - & - & - & - & - & + & - & + & 39 \\
\hline Dental anomalies & - & - & - & - & - & + & - & - & + & - & - & - & - & - & - & - & - & + & 17 \\
\hline
\end{tabular}

$\mathrm{ASD}=$ autism spectrum disorder, $\mathrm{dn}=$ de novo, $\mathrm{F}=$ female, $\mathrm{fs}=$ frameshift, $\mathrm{M}=$ male, $\mathrm{ms}=$ missense, $\mathrm{na}=$ not available, $\mathrm{ns}=$ nonsense, $\mathrm{p}=$ paternal, ss= splice site, + yes, - no. 


\section{Figures}

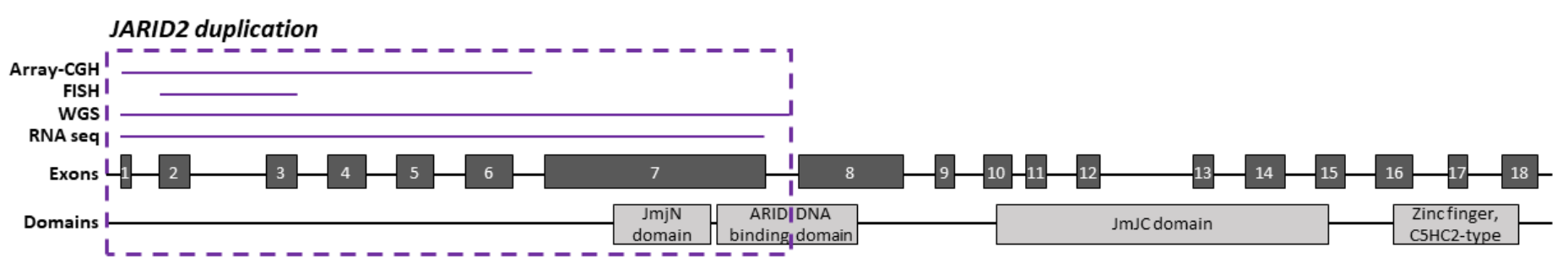

\section{Figure 1}

Figure 1. A schematic representation of JARID2 gene and the duplicated region. The partial JARID2 duplication of the index patient was detected using various methods including whole genome array-CGH (exons 1-6), fluorescence in situ hybridization (exons 2-3), whole genome sequencing (exons 1-7) and RNA sequencing (exons 1-7). The duplication covers the JmjN domain and part of the ARID/BRIGHT DNA binding domain. The figure is not in scale due to large introns. Detailed results of each method are presented in the Supplementary Data 1A-D and Supplementary Tables 1-3.

\section{Supplementary Files}

This is a list of supplementary files associated with this preprint. Click to download.

- SupplementaryData1.pdf

- SupplementaryTable1CNVresults.xlsx

- SupplementaryTable2QualityMetrics.xIsx

- SupplementaryTable3RNAseq.xIsx 